
SciDioc

International Journal of Reproduction, Fertility \& Sexual Health (IJRFSH)

ISSN: $2377-1887$

\title{
Validity of Ultrasound in diagnosis of Abnormal Placental Adherence at Third Trimester of Pregnancy
}

\author{
Case Report
}

Mawaldi L ${ }^{1 *}$, Sultan $\mathrm{S}^{1}$, Al Zahrani $\mathrm{M}^{1}$, Al Ghtani $\mathrm{N}^{1}$, Ahmed $\mathrm{AE}^{2}$

${ }^{1}$ Department of Gynecology and Obstetrics, King Abdulaziz Medical City, Ministry of National Guard, Riyadh, Kingdom of Saudi Arabia. ${ }^{2}$ College of Public Health and Health Informatics, King Saud Bin Abdulaziz University for Health Sciences, Riyadh, Kingdom of Saudi Arabia.

\section{Abstract}

Objective: To assess the value of ultrasound in diagnosis of abnormal adherent placenta in third trimester of pregnancy, which causes a life threatening maternal hemorrhage.

Methods: A retrospective cohort observational study of (68) patients in Ob/Gyn Department within five years, from the first of January 2009 to the last of December 2014 during their cesarean section found to be placenta accreta, increta, or perc reta at 28 weeks and above, and to compare the finding with their finding of ultrasound antenatally, and the histopathology after surgery.

Results: The surgery findings revealed that $49(72.1 \%)$ of the women had adherent placenta, $58(85.3 \%)$ had abnormal placentation by ultrasound, whereas only 10(14.7\%) normal. 36(52.9\%) confirmed by histopathology exam (15 acreta, 15 accreta increta, and 6 acreta precreta). The ultrasound findings revealed an area under the curve (AUC) of 53.8\%, sensitivity of $88.9 \%$, and specificity of only $18.8 \%$ while gave unsatisfactory agreement $(55.9 \%)$ with histopathology exam, ultrasound failed to detect normal findings in 26 cases.

Conclusion: The ultrasound examination is available, cheap, and easy to use, but it is not specifically reliable in diagnosis of abnormal adherent placenta in third trimester of pregnancy.

Keywords: Placenta Accrete; Increta; Percreta.

\section{Introduction}

Placenta accreta refers to an abnormality of placental implantation in which the anchoring placental villi attach to myometrium rather than decidua, resulting in a morbidly adherent placenta and massive hemorrhage. Placenta increta (chorionic villi penetrate into the myometrium) and placenta percreta (chorionic villi penetrate through the myometrium to the uterine serosa or adjacent organs). The pathogenesis is primarily attributed to defective decidualization of the implantation site [1-3]. The marked increase has been attributed due to increase the prevalence of cesarean delivery in recent years [4], or related to any previous uterine surgery, allows the placenta to attach directly to the myometrium [5, 6]. The frequency of placenta accreta increases with an increasing number of cesarean deliveries as follows [7, 8]: No previous cesarean birth 1- 5\%, One cesarean birth 11- $25 \%$, Two previous cesarean births $35-47 \%$, Three previous cesarean births $40 \%$, and Four or more previous cesarean births 50 - 67\% [9]. Other risk factors include a history of uterine surgery: myomectomy, removal of intrauterine adhesions, cornual resection of ectopic pregnancy, curettage, and maternal age greater than 35 years [10, 11]. The most common diagnostic image used for evaluating the placental position is trans-abdominal, and trans-vaginal ultrasound [12-15]. In the second and third trimesters [16, 17]: A loss of placental homogeneity, suggestive of placenta accreta which is replaced by intraplacental sonolucent spaces, and loss or thinning of the normal hypoechoic area behind the placenta, and loss or disruption of the normally continuous white line representing the bladder wall-uterine serosa interface, bulging of the placenta into the posterior wall of the bladder [18].

In a 2014 review and meta-analysis of 18 studies totaling 1010 pregnancies at risk for placenta accreta, magnetic resonance imaging (MRI) had high diagnostic accuracy for detection of placenta

*Corresponding Author:

Dr. Mawaldi L,

Department of gynecology and obstetrics, King abdulaziz medical city, Ministry of national guard, Riyadh- Kingdom of Saudi Arabia.

Tel: 00966502826134

E-mail: mawaldil@ngha.med.sa

Received: April 14, 2016

Accepted: May 18, 2016

Published: May 23, 2016

Citation: Mawaldi L, Sultan S, Al Zahrani M, Al Ghtani N, Ahmed AE (2016) Validity of Ultrasound in diagnosis of Abnormal Placental Adherence at Third Trimester of Pregnancy. Int J Reprod Fertil Sex Health, S1:003, 12-16. doi: http://dx.doi.org/10.19070/2377-1887-SI01003

Copyright: Mawaldi L ${ }^{\circ}$ 2016. This is an open-access article distributed under the terms of the Creative Commons Attribution License, which permits unrestricted use, distribution and reproduction in any medium, provided the original author and source are credited. 
accreta: sensitivity 94.4 percent (95\% CI 86.0-97.9), specificity 84 percent (95\% CI 76.0-89.8), positive likelihood ratio $5.91(95 \%$ CI 3.73-9.39), and negative likelihood ratio 0.07 (95\% CI $0.02-$ 0.18) [19]. MRI can be more useful than ultrasound in two clinical scenarios:1- Evaluation of a possible posterior placenta accreta because the bladder cannot be used to help clarify the placentalmyometriom interface, and 2- Assessment of the depth of myometriom, and parametriom involvement, and bladder involvement $[20,21]$. Our study will focus on the validity of ultrasound in diagnosis of adherent placenta in our department.

\section{Methods}

A retrospective cohort observational study of (68) patients in $\mathrm{Ob} / \mathrm{Gyn}$ Department within five years, from the first of January
2009 to the last of December 2014 during their cesarean section found to be placenta accreta, increta, or percreta at 28 weeks and above, comparing the finding with their finding of ultrasound antenatally, and the histopathology after surgery.

\section{Statistical Analysis}

The data were analyzed using IBM SPSS software V22. The association between abnormal surgery findings, and women characteristics was assessed using the Chi-square/Fisher's Exact test for categorical variables. The test findings were categorized into nor$\mathrm{mal}$ and abnormal (moderate-to-severe). Reference to histopathology examinations, the accuracy measurements for ultrasound, and surgery findings were evaluated by area under the curve, sensitivity, specificity, positive predictive value, negative predictive

Table 1. Abnormal surgery findings and patients' characteristics.

\begin{tabular}{|c|c|c|c|c|c|c|c|c|}
\hline \multirow[b]{2}{*}{ Characteristics } & & \multicolumn{2}{|c|}{$\begin{array}{c}\text { Overall } \\
N=68\end{array}$} & \multicolumn{2}{|c|}{$\begin{array}{c}\text { Abnormal } \\
49(72.1 \%)\end{array}$} & \multicolumn{3}{|c|}{$\begin{array}{c}\text { Normal } \\
19(27.9 \%)\end{array}$} \\
\hline & & $\mathbf{n}$ & $\%$ & $\mathbf{n}$ & $\%$ & $\mathbf{n}$ & $\%$ & $\mathbf{P}$ \\
\hline \multirow{2}{*}{ Gestational age } & $<37$ & 49 & 72.1 & 39 & 79.6 & 10 & 20.4 & \multirow{2}{*}{0.026} \\
\hline & $\geq 37$ & 19 & 27.9 & 10 & 52.6 & 9 & 47.4 & \\
\hline \multirow{2}{*}{ Age } & $<35$ & 26 & 38.2 & 19 & 73.1 & 7 & 26.9 & \multirow{2}{*}{0.883} \\
\hline & $\geq 35$ & 42 & 61.8 & 30 & 71.4 & 12 & 28.6 & \\
\hline \multirow{2}{*}{ No. Caesarean section } & $<3$ & \multirow{2}{*}{$\begin{array}{l}27 \\
41 \\
\end{array}$} & \multirow{2}{*}{$\begin{array}{l}39.7 \\
60.3\end{array}$} & \multirow{2}{*}{$\begin{array}{l}22 \\
27 \\
\end{array}$} & \multirow{2}{*}{$\begin{array}{l}81.5 \\
65.9 \\
\end{array}$} & 5 & 18.5 & 0.16 \\
\hline & $\geq 3$ & & & & & 14 & 34.1 & \\
\hline \multirow{2}{*}{ Previous uterine surgery } & Yes & \multirow{2}{*}{$\begin{array}{l}25 \\
43\end{array}$} & \multirow{2}{*}{$\begin{array}{l}36.8 \\
63.2\end{array}$} & \multirow{2}{*}{$\begin{array}{l}17 \\
32\end{array}$} & \multirow{2}{*}{$\begin{array}{c}68 \\
74.4\end{array}$} & 8 & 32 & 0.57 \\
\hline & No & & & & & 11 & 25.6 & \\
\hline \multirow{2}{*}{ Antenatal bleeding } & Yes & 36 & 52.9 & 29 & 80.6 & 7 & 19.4 & \multirow{2}{*}{0.098} \\
\hline & No & 32 & 47.1 & 20 & 62.5 & 12 & 37.5 & \\
\hline \multirow{2}{*}{ Emergency Bleeding } & Yes & 31 & 45.6 & 26 & 83.9 & 5 & 16.1 & \multirow{2}{*}{$0.047^{*}$} \\
\hline & No & 37 & 54.4 & 23 & 62.2 & 14 & 37.8 & \\
\hline \multirow{2}{*}{ Elective } & Yes & 37 & 54.4 & 23 & 62.2 & 14 & 37.8 & \multirow{2}{*}{$0.047^{*}$} \\
\hline & No & 31 & 45.6 & 26 & 83.9 & 5 & 16.1 & \\
\hline \multirow{2}{*}{ Management of placenta } & Yes & 35 & 51.5 & 34 & 97.1 & 1 & 2.9 & \multirow{2}{*}{0.001} \\
\hline & No & 33 & 48.5 & 15 & 45.5 & 18 & 54.5 & \\
\hline Hysterectomy & Yes & 29 & 42.6 & 28 & 96.6 & 1 & 3.4 & $0001^{*}$ \\
\hline Hysterectomy & No & 39 & 57.4 & 21 & 53.8 & 18 & 46.2 & 0.001 \\
\hline Bladder involvement & Yes & 16 & 23.5 & 16 & 100 & 0 & 0 & $0003^{*}$ \\
\hline Dradaer invorventent & No & 52 & 76.5 & 33 & 63.5 & 19 & 36.5 & 0.005 \\
\hline In lahor & Yes & 30 & 44.1 & 25 & 83.3 & 5 & 16.7 & 0066 \\
\hline In labor & No & 38 & 55.9 & 24 & 63.2 & 14 & 36.8 & 0.000 \\
\hline РPH_RT & Yes & 60 & 88.2 & 48 & 80 & 12 & 20 & $0 \cap 01 *$ \\
\hline 1 11-D1 & No & 8 & 11.8 & 1 & 12.5 & 7 & 87.5 & 0.001 \\
\hline ICU reguired & Yes & 16 & 23.5 & 15 & 93.8 & 1 & 6.3 & $0022^{*}$ \\
\hline ICU required & No & 52 & 76.5 & 34 & 65.4 & 18 & 34.6 & 0.049 \\
\hline uterine artery emboliza- & Yes & 5 & 7.4 & 5 & 100 & 0 & 0 & \\
\hline tion & No & 63 & 92.6 & 44 & 69.8 & 19 & 30.2 & 0.312 \\
\hline Internal iliac artery liga- & Yes & 20 & 29.4 & 18 & 90 & 2 & 10 & $0033^{*}$ \\
\hline tion & No & 48 & 70.6 & 31 & 64.6 & 17 & 35.4 & 0.053 \\
\hline Internal iliac artery bal- & Yes & 39 & 57.4 & 23 & 59 & 16 & 41 & $0005^{*}$ \\
\hline looning & No & 29 & 42.6 & 26 & 89.7 & 3 & 10.3 & 0.005 \\
\hline Ultrasound findinos & Abnormal & 58 & 85.3 & 39 & 67.2 & 19 & 32.8 & 0.052 \\
\hline & Normal & 10 & 14.7 & 10 & 100 & 0 & 0 & \\
\hline & Abnormal & 36 & 52.9 & 35 & 97.2 & 1 & 2.8 & \\
\hline Histopathology findings & Normal & 32 & 47.1 & 14 & 43.8 & 18 & 56.3 & 0.001 \\
\hline
\end{tabular}

Chi-square test/ Fisher's exact test is significant at $\alpha=0.05$. 
value, and agreement.

\section{Results}

A total of 68 women was our sample, the mean of age was 35.6 years ( \pm SD 3.9), between 23 to 45 years fulfilled the study criteria. The mean gestational age was 34.8 weeks ( \pm SD 2.6) with a range between 28 - 38 weeks. Of the sample, $61.8 \%$ were aged more than 35 years old, $72.1 \%$ had gestational age less than 37 weeks; $60.3 \%$ had three or more prior caesarean sections. Other characteristics are shown in Table 1.

The surgery findings revealed that $49(72.1 \%)$ of the women had abnormal placentation. Most women 58(85.3\%) had confirmed ultrasound results, whereas only $10(14.7 \%)$ had normal ultrasound results. $36(52.9 \%)$ of the women were classified as having abnormal results by histopathology examinations. Of the $36 \mathrm{ab}-$ normal findings, histopathology examinations revealed 15 acreta, 15 acreta increta, and 6 acreta precreta.

Subgroup analyses were performed using Chi-square/Fisher's Exact test Table 1. Percentage of abnormal placentation in surgery finding was higher in women with gestational age less than 37 weeks than women with gestational age 37 weeks or more $(79.6 \%$ vs $52.6 \%, \mathrm{P}=0.026)$. Percentage of abnormal placentation in surgery finding significantly increased with bleeding emergency (83.9\% vs $62.2 \%, \mathrm{P}=0.047$ ). Extra management required to remove the placenta with the group of abnormal placentation in surgery $(97.1 \%$ vs $45.5 \%, \mathrm{P}=0.001)$. Hysterectomy was required in the group of abnormal placentation ( $96.6 \%$ vs $53.8 \%, \mathrm{P}=0.001)$. Internal iliac artery ballooning performed in 39 cases $(\mathrm{P}=0.005)$, and internal iliac artery ligation in 20 cases $(\mathrm{P}=0.033)$, whereas uterine artery embolization were performed in 5 cases only.

Bladder involvement in the group of abnormal placentation in surgery, was observed in $(100 \%)$ compared to $(63.5 \%)$ in normal group $(\mathrm{P}=0.003)$. Postpartum hemorrhage, and blood transfusion were observed in the group of abnormal placentation in surgery, compared with normal ( $80 \%$ vs $12.5 \%, \mathrm{P}=0.001)$. ICU admission required in the group of abnormal placentation in surgery $(93.8 \%$ vs $65.4 \%$ in normal, $\mathrm{P}=0.029)$.

\section{Discussion}

The area under the curve, sensitivity, specificity, positive predictive value (PPV), negative predictive value (NPV), and the agreement for ultrasound, and surgery findings reference to histopathology examinations are shown in Table 2 . The ultrasound findings revealed an area under the curve (AUC) of $53.8 \%$, sensitivity of $88.9 \%$, and specificity of only $18.8 \%$ while gave unsatisfactory agreement $(55.9 \%)$ with histopathology examination.

The surgery findings tend to have better discriminative ability than ultrasound findings with an area under the curve (AUC) of $76.7 \%$, sensitivity $97.22 \%$, and specificity $56.25 \%$ while gave satisfactory agreement $(78 \%)$ with histopathology examination. Furthermore, the ultrasound and surgery findings were assessed by the receiver-operating characteristic (ROC) curve in Figure 1. revealed the surgery was better than ultrasound in detecting abnormal placentation.

In Table3 : The ultrasound reference to surgery findings, revealed an area under the curve (AUC) of $60.2 \%$, sensitivity of $79.6 \%$, and specificity of only $0.0 \%$, positive predictive value (PPV)

Table 2. Accuracy measurements for ultrasound and surgery findings reference to Histopathology Findings.

\begin{tabular}{|c|c|c|}
\hline & Ultrasound findings & Surgery findings \\
\hline \multicolumn{3}{|l|}{ Accuracy } \\
\hline AUC $(95 \% \mathrm{CI})$ & $53.8 \%(40.0 \%-67.7 \%)$ & $76.7 \%(64.8 \%-88.6 \%)$ \\
\hline Sensitivity $(95 \% \mathrm{CI})$ & $88.9 \%(73.9 \%-96.9 \%)$ & $97.22 \%(85.5 \%-99.9 \%)$ \\
\hline Specificity $(95 \% \mathrm{CI})$ & $18.8 \%(7.2 \%-36.4 \%)$ & $56.25 \%(37.7 \%-73.6 \%)$ \\
\hline PPV $(95 \% \mathrm{CI})$ & $55.2 \%(41.5 \%-68.3 \%)$ & $71.43 \%(56.7 \%-83.4 \%)$ \\
\hline $\mathrm{NPV}(95 \% \mathrm{CI})$ & $60.0 \%(26.2 \%-87.8 \%)$ & $94.74 \%(74.0 \%-99.9 \%)$ \\
\hline \multicolumn{3}{|l|}{ Agreement } \\
\hline Overall agreement $\%$ & $55.90 \%$ & $78.0 \%$ \\
\hline Kappa (P-value) & $0.09(\mathrm{P}$-value $=0.375)$ & $0.55(\mathrm{P}$-value $=0.001)$ \\
\hline
\end{tabular}

AUC $=$ Area Under the Curve; PPV $=$ Positive Predictive Value; NPV Negative Predictive Value

Table 3. Accuracy measurements for ultrasound reference to surgery findings.

\begin{tabular}{|c|c|}
\hline & Ultrasound findings \\
\hline Accuracy & \\
\hline AUC $(95 \% \mathrm{CI})$ & $60.2 \%(46.3 \%-74.1 \%)$ \\
\hline Sensitivity $(95 \% \mathrm{CI})$ & $79.6 \%(65.7 \%-89.8 \%)$ \\
\hline Specificity $(95 \% \mathrm{CI})$ & $0 \%(0.0 \%-17.7 \%)$ \\
\hline PPV $(95 \% \mathrm{CI})$ & $67.2 \%(53.7 \%-79.0 \%)$ \\
\hline NPV $(95 \% \mathrm{CI})$ & $0.0 \%(0.0 \%-30.9 \%)$ \\
\hline Agreement & \\
\hline Overall agreement $\%$ & $57.40 \%$ \\
\hline Kappa (P-value) & $-0.2(\mathrm{P}$-value $=0.033)$ \\
\hline
\end{tabular}

AUC $=$ Area Under the Curve; PPV $=$ Positive Predictive Value; NPV Negative Predictive Value 
$67.2 \%$, and negative predictive value (NPV) $0.0 \%$, with unsatisfactory agreement $57.4 \%$. So the ultrasound is not a specific medical image reliable for diagnosis of abnormal placental adherence at third trimester of pregnancy, could be because of over diagnosis report of positive finding. In Table 4: Ultrasound findings vs Surgery findings Cross-tabulation, revealed 39 cases reported by ultrasound as abnormal and confirmed by surgery (True positive), while 19 cases counted as abnormal by ultrasound and found to be normal during surgery (False positive), 10 cases reported as normal by ultrasound found to be abnormal during surgery (False negative), none of the cases found to be normal during surgery (True negative).
In Table 5: Ultrasound findings vs Histopathology findings Cross-tabulation. Of 32 normal cases, ultrasound failed to detect normal findings in 26 cases. In Table 6: Surgery findings vs Histopathology findings Cross-tabulation, 14 normal cases from 32 normal cases were counted as abnormal during surgery, could be due to difficulty during removal of placenta.

\section{Conclusion}

The ultrasound examination is available, cheap, and easy to use, but it is not specifically reliable in diagnosis of abnormal adherent placenta in third trimester of pregnancy.

Figure 1. Receiver-operating characteristic (ROC) curve for ultrasound and surgery findings.

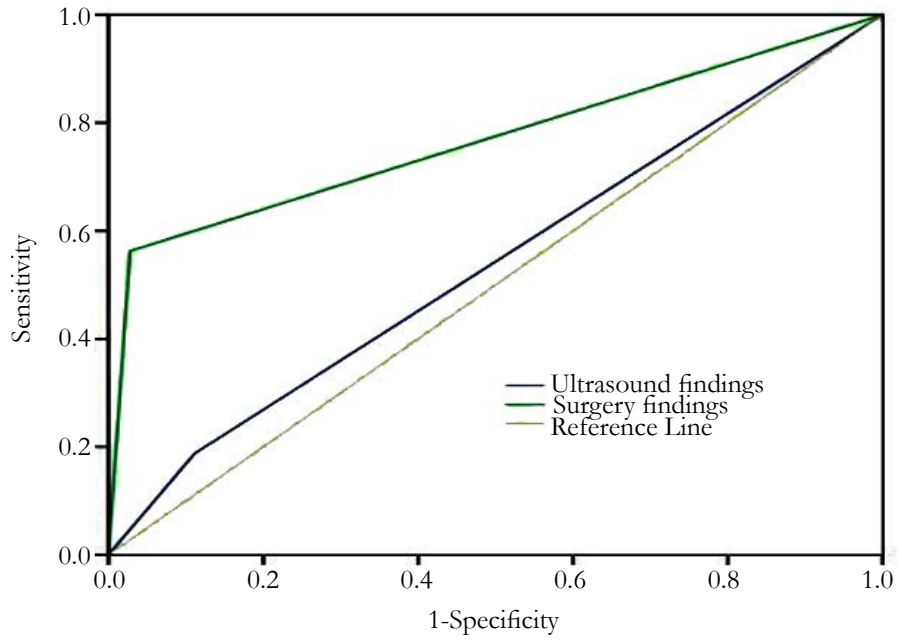

Table 4. Ultrasound findings * Surgery findings Cross-tabulation.

\begin{tabular}{|c|c|c|c|c|c|}
\hline & \multicolumn{2}{|c|}{ Surgery findings } & \multirow{2}{*}{ Total } \\
\hline & & & Abnormal & Normal & \\
\hline \multirow{2}{*}{$\begin{array}{l}\text { Ultrasound } \\
\text { findings }\end{array}$} & Abnormal & $\begin{array}{c}\text { Count } \\
\% \text { within Surgery findings }\end{array}$ & $\begin{array}{l}\mathrm{TP}=39 \\
79.6 \%\end{array}$ & $\begin{array}{l}\mathrm{FP}=19 \\
100.0 \%\end{array}$ & $\begin{array}{c}58 \\
85.3 \%\end{array}$ \\
\hline & Normal & $\begin{array}{c}\text { Count } \\
\% \text { within Surgery findings }\end{array}$ & $\begin{array}{c}\mathrm{FN}=10 \\
20.4 \%\end{array}$ & $\begin{array}{l}\mathrm{TN}=0 \\
0.0 \%\end{array}$ & $\begin{array}{c}10 \\
14.7 \%\end{array}$ \\
\hline \multirow{2}{*}{ Total } & & Count & 49 & 19 & 68 \\
\hline & \multicolumn{2}{|c|}{$\%$ within Surgery findings } & $100.0 \%$ & $100.0 \%$ & $100.0 \%$ \\
\hline
\end{tabular}

Table 5. Ultrasound findings * Histopathology findings Cross-tabulation.

\begin{tabular}{|c|c|c|c|c|c|}
\hline & \multicolumn{2}{|c|}{$\begin{array}{l}\text { Histopathology } \\
\text { findings }\end{array}$} & \multirow[t]{2}{*}{ Total } \\
\hline & & & Abnormal & Normal & \\
\hline \multirow{2}{*}{$\begin{array}{l}\text { Ultrasound } \\
\text { findings }\end{array}$} & Abnormal & $\begin{array}{c}\text { Count } \\
\% \text { within Histopathology } \\
\text { findings }\end{array}$ & $\begin{array}{c}32 \\
88.90 \%\end{array}$ & $\begin{array}{c}26 \\
81.30 \%\end{array}$ & $\begin{array}{c}58 \\
85.30 \%\end{array}$ \\
\hline & Normal & $\begin{array}{c}\text { Count } \\
\% \text { within Histopathology } \\
\text { findings }\end{array}$ & $\begin{array}{c}4 \\
11.10 \%\end{array}$ & $\begin{array}{c}6 \\
18.80 \%\end{array}$ & $\begin{array}{c}10 \\
14.70 \%\end{array}$ \\
\hline \multirow{2}{*}{ Total } & & Count & 36 & 32 & 68 \\
\hline & \multicolumn{2}{|c|}{ \% within Histopathology findings } & $100.00 \%$ & $100.00 \%$ & $100.00 \%$ \\
\hline
\end{tabular}


Table 6. Surgery findings * Histopathology findings Cross-tabulation.

\begin{tabular}{|c|c|c|c|c|c|}
\hline & & & \multicolumn{2}{|c|}{$\begin{array}{c}\text { Histopathology } \\
\text { findings }\end{array}$} & \multirow[t]{2}{*}{ Total } \\
\hline & & & Abnormal & Normal & \\
\hline \multirow[t]{2}{*}{$\begin{array}{l}\text { Surgery } \\
\text { findings }\end{array}$} & Abnormal & $\begin{array}{l}\text { Count } \\
\% \text { within Histopathology } \\
\text { findings }\end{array}$ & $\begin{array}{c}35 \\
97.20 \%\end{array}$ & $\begin{array}{c}14 \\
43.80 \%\end{array}$ & $\begin{array}{c}49 \\
72.10 \%\end{array}$ \\
\hline & Normal & $\begin{array}{l}\text { Count } \\
\% \text { within Histopathology } \\
\text { findings }\end{array}$ & $\begin{array}{c}1 \\
2.80 \%\end{array}$ & $\begin{array}{c}18 \\
56.30 \%\end{array}$ & $\begin{array}{c}19 \\
27.90 \%\end{array}$ \\
\hline \multirow[t]{2}{*}{ Total } & & Count & 36 & 32 & 68 \\
\hline & \multicolumn{2}{|c|}{$\%$ within Histopathology findings } & $100.00 \%$ & $100.00 \%$ & $100.00 \%$ \\
\hline
\end{tabular}

\section{References}

[1]. Tantbirojn P, Crum CP, Parast MM (2008) Pathophysiology of placenta creta: the role of decidua and extravillous trophoblast. Placenta 29(7): 639645

[2]. Miller DA, Chollet JA, Goodwin TM (1997) Clinical risk factors for placenta previa-placenta accreta. Am J Obstet Gynecol 177(1): 210-214.

[3]. Wu S, Kocherginsky M, Hibbard JU (2005) Abnormal placentation: twentyyear analysis. Am J Obstet Gynecol 192(5): 1458-1461.

[4]. Read JA, Cotton DB, Miller FC (1980) Placenta accreta: changing clinical aspects and outcome. Obstet Gynecol 56(1): 31-34.

[5]. Khong TY. The pathology of placenta accreta, a worldwide epidemic. J Clin Pathol 2008; 61:1243.

[6]. Jauniaux E, Jurkovic D (2012) Placenta accreta: pathogenesis of a $20^{\text {th }}$ century iatrogenic uterine disease. Placenta 33(4): 244-251.

[7]. Clark SL, Koonings PP, Phelan JP (1985) Placenta previa/accreta and prior cesarean section. Obstet Gynecol 66(1): 89-92.

[8]. Silver RM, Landon MB, Rouse DJ, et al. (2006) Maternal morbidity associated with multiple repeat cesarean deliveries. Obstet Gynecol 107(6): 1226-1232.

[9]. Cunningham FG, Bangdiwala S, Brown SS, Dean TM, Frederiksen M, et al. (2010) National Institutes of Health Consensus Development Conference Statement: Vaginal Birth After Cesarean: New Insights March 8-10, 2010. Obstetrics \& Gynecology 115(6): 1279-1295.

[10]. Nageotte MP (2014) Always be vigilant for placenta accreta. Am J Obstet Gynecol 211(2): 87-88.

[11]. Timor-Tritsch IE, Monteagudo A, Cali G, et al. (2014) Cesarean scar pregnancy is a precursor of morbidly adherent placenta. Ultrasound Obstet Gynecol 44(3): 346-353.

[12]. Comstock CH (2005) Antenatal diagnosis of placenta accreta: a review. Ultrasound Obstet Gynecol 26(1): 89-96.

[13]. Warshak CR, Eskander R, Hull AD, et al. (2006) Accuracy of ultrasonography and magnetic resonance imaging in the diagnosis of placenta accreta. Obstet Gynecol 108(1): 573-581.
[14]. D'Antonio F, Iacovella C, Bhide A (2013) Prenatal identification of invasive placentation using ultrasound: systematic review and meta-analysis. Ultrasound Obstet Gynecol 42(5): 509-517.

[15]. Bowman ZS, Eller AG, Kennedy AM, et al. (2014) Accuracy of ultrasound for the prediction of placenta accreta. Am J Obstet Gynecol 211(12): 177. e1-7.

[16]. Finberg HJ, Williams JW (1992) Placenta accreta: prospective sonographic diagnosis in patients with placenta previa and prior cesarean section. J Ultrasound Med 11(7): 333-343.

[17]. Guy GP, Peisner DB, Timor-Tritsch IE (1990) Ultrasonographic evaluation of uteroplacental blood flow patterns of abnormally located and adherent placentas. Am J Obstet Gynecol 163(3): 723-727.

[18]. Ballas J, Pretorius D, Hull AD, et al. (2012) Identifying sonographic markers for placenta accreta in the first trimester. J Ultrasound Med 31(11): 1835-1841.

[19]. D'Antonio F, Iacovella C, Palacios-Jaraquemada J, et al. (2014) Prenatal identification of invasive placentation using magnetic resonance imaging: systematic review and meta-analysis. Ultrasound Obstet Gynecol 44(1): 8-16.

[20]. Maldjian C, Adam R, Pelosi M, et al. (1999) MRI appearance of placenta percreta and placenta accreta. Magn Reson Imaging 17(7): 965-971.

[21]. Kirkinen P, Helin-Martikainen HL, Vanninen R, Partanen K (1998) Placenta accreta: imaging by gray-scale and contrast-enhanced color Doppler sonography and magnetic resonance imaging. J Clin Ultrasound 26(2): 90-94.

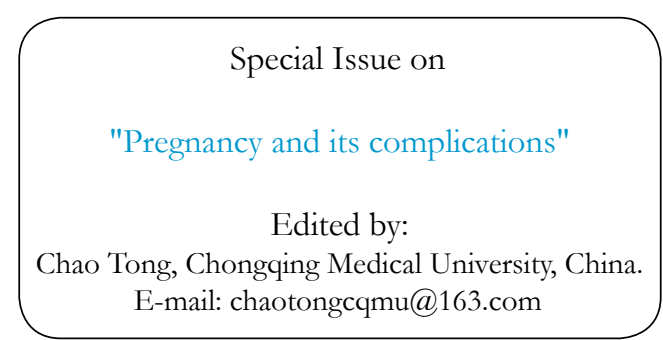

